\title{
MOCVD-grown indium phosphide nanowires for optoelectronics
}

\begin{abstract}
We demonstrate how growth parameters may be adopted to produce morphologically controlled high-quality indium phosphide (InP) nanowires suitable for optoelectronic device applications. Growth temperature, V/III ratio, and catalyst particle size have a significant effect on the morphology, crystallographic quality, and optical properties of the resulting nanowires. Significantly, we find that higher growth temperatures or higher V/III ratios promote the formation of wurtzite (WZ) nanowires while zinc-blende (ZB) nanowires are favourable at lower growth temperatures and lower V/III ratios. Results also show that InP nanowires grow preferably in the WZ crystal structure than the ZB crystal structure with increasing V/III ratio or decreasing diameter. This causes a blue-shift in the bandgap as growth temperature increases. These results show that careful control of growth temperature, V/III ratio and catalyst size are crucial for obtaining InP nanowires of a specific crystal structure needed for device applications.
\end{abstract}

Keyword: InP; Nanowires; V/III ratio; Wurtzite; Zinc-blende 